\title{
Why are the Acta Neuropathologica so fast, with a mean time from submission to first decision of 16 days?
}

\author{
Werner Paulus
}

Received: 8 January 2008 / Published online: 31 January 2008

(C) Springer-Verlag 2008

As an author, there is almost nothing in the publication business that bothers me more than that of the delayed editorial decisions. I often felt annoyed or I despaired when a journal did not respond for 4 or 6 months after I had submitted a paper. I felt that editors and referees should recall that a scientific paper is not only the final result of hard work, but is kind of a baby with some of the corresponding emotional implications, and that careless treatment by a journal represents an offence towards authors. In fact, one major reason why I became an editor was my own negative experience with tardy journals.

I am therefore happy in this regard that the Acta Neuropathologica perform better than most other journals. During the past 3 years, the time from submission to first decision has been around 17 days for original submissions, and between 2 and 8 days for revised versions (Table 1). It has been encouraging to learn from authors that our fast turnaround time has been noticed increasingly and has been much appreciated. Because data on decision times are usually not published and thus not generally available, there is not much pressure on editors to become faster. If rankings of editorial speed were published (like impact factors), effort and efficiency of many journals would certainly increase and the service to authors would improve. Since most journals use similar or identical online submission systems where metadata can easily be retrieved, a transparent comparison of decision times is feasible.

The major reason for our fast decision time is the dedication and hard work of our referees. In 2007, they submitted

W. Paulus $(\square)$

Institute of Neuropathology, University Hospital,

Domagkstrasse 19, 48149 Münster, Germany

e-mail: paulusw@uni-muenster.de;

werner.paulus@uni-muenster.de reviews within a mean time of 11 days after agreeing to review. This accomplishment is related to careful selection of referees. The first experts who come to mind after reading a submission are often not the most efficient referees. For each manuscript, I perform a PubMed search and then I screen websites of institutions to identify colleagues who might have the greatest interest in this paper. Indicators that correlate with excellent review performance include previous review activities for Acta (frequencies of accept vs. decline to review, speed and quality of reviews) and academic position (Chairmen tend to be overcommitted), among others. Some simple measures can considerably reduce review times, such as verification of e-mail addresses via websites, and a tight first response time (for accept or decline to review) of 3 days. A remarkable $64 \%$ of invited referees have accepted to deliver within 14 days, while the remaining $36 \%$ declined or did not respond. In addition to external referees, the Editorial Board of the Acta consists of particularly dedicated experts who are willing to review up to 12 papers per year, each of them within 14 days. Editorial Board members are also expected to review occasional papers lying somewhat outside of their own area of research, but for which no other suitable external referees could be identified within a week or so. Furthermore, we are pertinacious in reminding late referees. If several reminders for 10 days have not been successful, we usually contact the referee by telephone. If we do not expect the review soon, we terminate this review, which has been necessary in only four cases in 2007. The longest time in 2007 for receiving a review was 39 days. I am always much impressed about the high motivation and enthusiasm of our referees.

Additional reasons of fast decision include short times from submission to editor assignment ( 0.2 days), and from editor assignment to reviewer invitation ( 0.5 days); a 
Table 1 Mean time from submission to decision (days)

\begin{tabular}{lrrr}
\hline & 2005 & 2006 & 2007 \\
\hline Original version & 17.2 & 17.5 & 15.7 \\
First revision & 6.8 & 7.4 & 7.9 \\
Second revision & 1.8 & 3.6 & 4.2 \\
\hline
\end{tabular}

simple structure of the Editorial Board without multiple levels of decision; and instantaneous beginning of checking for formal accuracy (format of references, resolution of figures, etc.) in parallel to the initiation of the review process so that no delay is introduced.

Interestingly, the time to decision may even be too fast for some authors. In 2007, $15 \%$ of manuscripts submitted to Acta Neuropathologica were rejected without consulting referees, for example, because they lacked novelty or because the subject was unrelated to neuropathology. This negative decision can often be made on the day of submission. Usually, the act of submitting a manuscript is the final outcome of laborious scientific work, writing and formatting, and provides authors with a good deal of relief and satisfaction. A negative decision within $2 \mathrm{~h}$ impairs this good feeling (especially if the author is still online). Furthermore, I have received letters from authors stating that ultrafast decisions are unfair, because a manuscript cannot be evaluated carefully enough. On the other hand, it seems fair to both authors and referees to reject an unacceptable paper straightaway.

Authors would like to receive not only speedy reviews but also fair and careful reviews. The question is, therefore, whether speed and quality of reviews are related. Because in this journal all reviews are rated from 0 (worst) to 100 (best), we plotted speed versus quality. As reflected in Fig. 1, there is no indication that review time is related to the quality of a review.

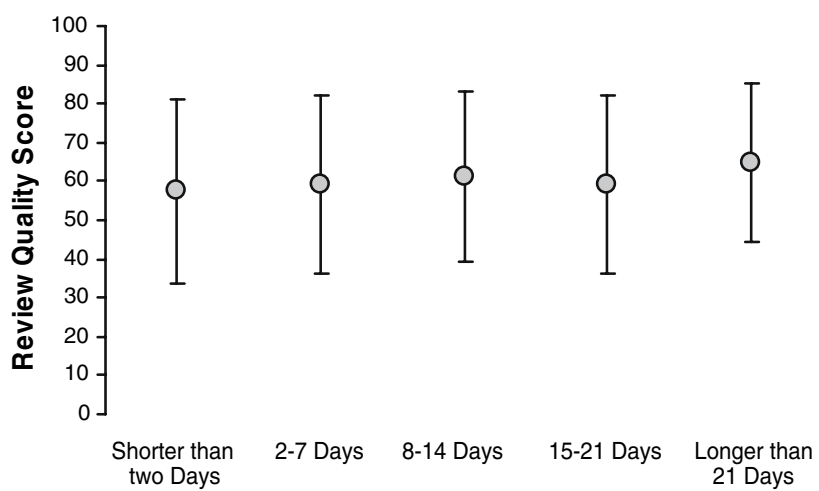

Fig. 1 Review quality score, shown as mean $\pm S D$, is not related to time of review (based on a total of 680 reviews performed in 2007)

I should also mention that the Acta are fast in other respects as well. Springer Publishers have organized production such that the time between acceptance and sending out proofs is only a few days, and the time between acceptance and online publication is about 2 weeks. Accordingly, online publication is possible within 30 days following the submission. An example is the study by Neumann et al. on TDP-43 (Acta Neuropathol 113: 543-548, 2007) for which the interval between submission and first decision was 12 days, for revision by the authors was 1 day, for resubmission to final decision was 0 days, and from final acceptance to publication in PubMed was 17 days.

To summarize, making speedy and careful decisions is quite easy for an editor, but only with the support of dedicated referees, with an enthusiastic Editorial Board and with an efficient editorial office. We are aware that speed of editorial decision is just one, but an important indicator of journal quality. We are working hard to maintain and improve all aspects of quality to make Acta Neuropathologica an even better journal. Please let me know if you have any ideas and suggestions or if you would like to contribute. 